\title{
Pigmented villonodular synovitis: a crowdsourcing study of two hundred and seventy two patients
}

\author{
Lizz van der Heijden $^{1}$ • Sheila R. Piner ${ }^{2}$ - Michiel Adrianus Josephus van de Sande ${ }^{1}$
}

Received: 14 December 2015 / Accepted: 19 April 2016/Published online: 12 May 2016

(C) The Author(s) 2016. This article is published with open access at Springerlink.com

\begin{abstract}
Purpose We aimed to ascertain the feasibility of crowdsourcing via Facebook for medical research purposes; by investigating surgical, oncological and functional outcome and quality-of-life (QOL) in patients with pigmented villonodular synovitis (PVNS) enrolled in a Facebook community (1112 members).

Methods Patients completed online open surveys on demographics, surgery and clinical outcomes (group 1); and patient-reported outcome measures (PROMs) including knee-injury osteoarthritis outcome score (KOOS), hipdisability osteoarthritis outcome score (HOOS), Toronto extremity salvage score (TESS) and SF-36 (group 2). Mean follow-up was 70 months (12-374). Consistency checks were performed with Cohen's kappa statistic for intra-rater agreement.

Results The first survey was completed by 272 patients (group 1) and 72 patients completed the second (group 2). In group 1, recurrence-rate was $58 \%$ (69/118) after arthroscopic, $36 \%(35 / 97)$ after open and $50 \%(5 / 10)$ after combined synovectomy $(p=0.003)$. In group 2 , recurrence-rate was $67 \%(26 / 39)$ after arthroscopic and $51 \%(17 / 33)$ after open synovectomy $(p=0.19)$. Recurrence-risk was increased for diffuse disease $(\mathrm{OR}=16 ; 95 \% \mathrm{CI}=3.2-85 ; p<0.001)$. Mean function
\end{abstract}

Lizz van der Heijden

lvanderheijden@lumc.nl

1 Orthopaedic Surgery, Leiden University Medical Centre, Postzone J11-R70, PO Box 9600, 2300 RC Leiden, The Netherlands

2 Leiden University Medical Centre, Postzone J11-R70, PO Box 9600, 2300 RC Leiden, The Netherlands and QOL did not differ after arthroscopic or open synovectomy: KOOS $49 v s .58(p=0.24)$, HOOS $62 v s$. $53(p=0.56)$, TESS 78 vs. $82(p=0.86)$, SF-36 $61 v s .66$ $(p=0.41)$. Cohen's kappa statistic for intra-rater agreement was good to outstanding ( $\mathrm{K}=0.68-0.95 ; p<0.001)$. Conclusion Local recurrence-risk was higher for diffusetype disease and arthroscopic synovectomy. Functional outcome and QOL were comparable for both types of surgery. Gathering data via crowdsourcing seems a promising and innovative way of evaluating rare diseases including PVNS.

Keywords Crowdsourcing - Diffuse-type giant cell tumor . Facebook $\cdot$ Functional outcome $\cdot$ Pigmented villonodular synovitis $\cdot$ PVNS $\cdot$ Quality of life

\section{Introduction}

Pigmented villonodular synovitis (PVNS) is a rare benign but locally aggressive giant cell tumour deriving from synovium, either localized giant cell tumour of tendon sheath (GCT-TS) or diffuse-type giant cell tumour (DtGCT) $[1,2]$. It is most commonly found in the knee (75\%; Fig. 1) and hip (15\%) [1, 3]. Treatment may consist of arthroscopic or open synovectomy, intraarticular radioactive colloids, radiation therapy or systemic targeted therapy $[4,5]$.

Rare diseases, including PVNS, are difficult to investigate as published case series are generally small, of retrospective nature and often describe research periods of several decades with a short follow-up duration. Therefore, these studies only provide levels of evidence 

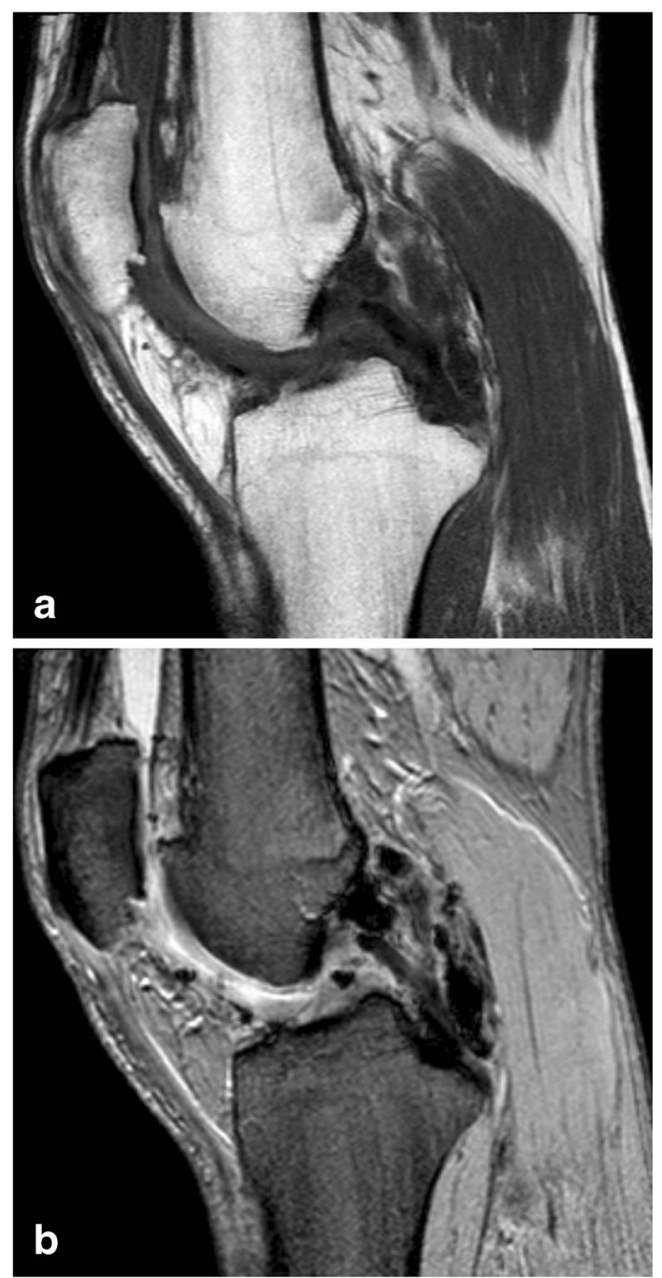

Fig. 1 MR images of a 27 year old male patient with recurrent diffuse PVNS in the knee after previous anterior arthroscopic synovectomy and ${ }^{90}$ Yttrium instillation show multiple intra-articular lesions dorsal to the posterior cruciate ligament, with low signal intensity on T1-weighted sequences (A) and heterogeneous intermediate signal intensity on T2weighted sequences (B) with artefacts due to haemosiderin depositions being characteristic for PVNS.

III-IV, and clear evidence is lacking as meta-analysis of gathered data is often not warranted.

With a growing number of patients using social media as a source of medical information, online patient platforms can also be used as a powerful educational tool. Gathering patient data via crowdsourcing on social media can be especially promising and useful in the evaluation of rare diseases, as large study populations may become easily available to researchers. Crowdsourcing is the practice of obtaining services, ideas or content by collecting contributions from a large group of people from an online community rather than from traditional data suppliers. This is already increasingly being used for meeting the needs of consumers, and we wanted to investigate if it could be used to evaluate patient satisfaction, functional outcome and quality of life for patients with PVNS. Crowdsourcing to obtain big data can be useful in all fields of medicine, especially regarding the evaluation of rare diseases and patient reported outcome measures (PROMs). Open online surveys may also be more attractive and quick to complete for the modern patient than conventional questionnaires on paper via regular mail.

Several small communities of patients with PVNS exist on Facebook; however, there is only one community with over 1100 patients at the time of study ( $>2000$ at the time of writing). On this forum, patients share information on their disease, experiences with treating physicians, surgery or other treatments and clinical outcome, and seek support from fellow patients. From this forum, we learned that adequate patient information on PVNS is deficient and that there is a desire among patients to interact with other patients and professionals and a growing demand for taking part in scientific research projects on PVNS. This is also reflected by recent initiatives such as PatientsLikeMe (www.patientslikeme.com), in which patients can track their outcomes by sharing their data and by participating in the design and implementation of patient-centred trials $[6,7]$. Such online patient communities may allow researchers rapid access to large study populations that are otherwise hard to assemble in rare diseases.

The aim of this study was to investigate whether we could use crowdsourcing via Facebook and online surveys for medical research purposes on PVNS. More specifically, we set out to correlate functional outcome and quality of life (QOL) to patient characteristics, surgical procedures and oncological outcome in patients with PVNS who were enrolled in a patient community on Facebook.

\section{Materials and methods}

In this open survey observational study, patients were recruited to complete questionnaires on their disease through a patient-initiated campaign on a PVNS patient community on Facebook (1112 members at time of consulting; https://www.facebook.com/groups/ $91851410592 /$ ?ref=ts\&fref=ts). A first online survey on demographics, clinical presentation, findings on imaging and biopsy material, type and localization of disease, surgical and adjuvant treatment, local recurrences, postoperative complications, functional outcome, quality of life (QOL) and follow-up was open from December 2012 to April 2014 (group 1). A second online survey using standardized and validated questionnaires on 
patient-reported outcome measures (PROMs) [8], to evaluate functional outcome and QOL, was open from August 2013 to February 2014 (group 2). The latter included range of motion (ROM), knee injury and osteoarthritis outcome score (KOOS) [9, 10], hip disability and osteoarthritis outcome score (HOOS) [11], Toronto extremity salvage score (TESS) [12] and short form-36 health survey (SF-36) [13]. Mean follow-up after index surgery was 70 months (range 12-374). The surveys were developed via SurveyMonkey® (https://www.surveymonkey.com) and usability and technical functionality were tested by the researchers. The surveys were announced on the Facebook community wall with a first notice of the study, and repeated requests for compilation after one, two, five, six and ten months. It was a voluntary survey and no incentives were offered. All responses were anonymous and automatically captured into an SPSS 20. 0 file, which was password protected and only accessible to the researchers. Completeness checks were performed through JAVAScript and only fully completed surveys were included in the analysis. Respondents were able to review and change their answers before submitting. Unique site visitors were determined by IP addresses, and in case of duplicate entries only the most recent ones were included in the analysis. Propensity scores to adjust for a non-representative sample were not used in this study, but results were compared with previous results from the literature. The checklist for Reporting Results of Internet E-Surveys (CHERRIES) was used (Appendix 1) $[14,15]$. None of the patients were recalled for this study. In the present study, data were not verified by contacting treating physicians or patients. By completing the surveys, patients gave their informed consent for this study. All procedures performed were in accordance with the ethical standards of Dutch law (Medical Research involving Human Subjects Act) and with the 1964 Helsinki declaration and its later amendments. For this type of study formal consent is not required.

\section{Statistical analysis}

Differences in dichotomous data were calculated with Chi-squared and Fisher's exact tests and in numerical data with Mann-Whitney U tests. Multivariable logistic regression analyses were performed to identify factors of influence on recurrence, including diffuse or localized disease, localization and type of surgery. To determine whether the results of this study are reliable, we performed consistency tests by evaluating intra-rater agreement for patients' answers between both online surveys. We used Cohen's kappa statistic to perform consistency checks by determining intra-rater agreement for patients that could be cross-linked by IP address in the two online surveys. Outstanding agreement was defined as $k>0.80$; substantial agreement as $K=0.60-0.79$; moderate agreement as $k=0.40-0.59$; and poor agreement as $k<0.40$ [16]. Data from the two surveys were not combined but analysed separately; hence, the occurrence of repeated measures was not accounted for. We used SPSS 20.0 (SPSS Inc, Armonk, NJ, USA) for statistical analyses. The level of statistical significance was set at $p<0.05$.

\section{Results}

All 1112 members of the PVNS patient community on Facebook, at the time of consulting, could view the online open survey. Participation rate was $26.3 \%$ (293/ $1112)$ in the first survey; and $11.8 \%(131 / 1112)$ for SF-36, $5.3 \%$ (59/1112) for KOOS, $0.8 \%$ (9/1112) for HOOS and $3.1 \%(34 / 1112)$ for TESS in the second survey. Completion rate was $92.8 \%(272 / 293)$ in the first survey; and $84.7 \%(111 / 131)$ for SF-36, $86.4 \%$ $(51 / 59)$ for KOOS, $100 \%$ (9/9) for KOOS and $100 \%$ (34/34) for TESS in the second survey.

From group 1, 272 patients were included (Table 1). They originated from 23 countries and underwent arthroscopic or open synovectomy between 1982 and 2012. Primary surgery was arthroscopic synovectomy $(n=118)$, open synovectomy $(n=97)$, combined arthroscopic and open synovectomy (hybrid synovectomy; $n=10)$ or unknown $(n=47)$. One hundred twenty two patients reported at least one local recurrence, 124 patients had no recurrences (Table 2). Mean number of surgeries was $2.6(1-9)$. Final surgery was open synovectomy $(n=52)$, arthroscopic synovectomy $(n=36)$, hybrid synovectomy $(n=10)$ or unknown $(n=24)$. Total hip arthroplasty was required in $13 / 25$ and total knee arthroplasty in 17/199 $(p<0.001)$. Adjuvant treatment was radiation therapy $(n=30)$, radioactive colloid instillation with ${ }^{90}$ Yttrium $(n=18)$, MCSFR targeted tyrosine kinase inhibitor imatinib $(n=8)$ or nilotinib $(n=2)$, cryosurgery $(n=3)$ or methotrexate $(n=1)$.

From group 2, 72 patients with PVNS in the knee or hip joint and a minimum follow-up of one year were included (Table 1). The knee was affected in 64 patients (52 diffuse; 12 localized) and the hip in eight (six diffuse; two localized). Other localizations $(n=18$; e.g. ankle, elbow and shoulder) were excluded from PROMs analyses, as we wanted to focus on the two most common localizations; however, they were used for consis- 
Table 1 Patient, tumour and treatment characteristics

\begin{tabular}{|c|c|c|c|c|}
\hline & \multicolumn{2}{|c|}{ Group $1(n=272)$} & \multicolumn{2}{|c|}{ Group $2(n=72)$} \\
\hline & mean & range & mean & range \\
\hline Age (years) & 32 & $11-67$ & 31 & $15-58$ \\
\hline Follow-up (months) & $\mathrm{U}$ & $\mathrm{U}$ & 68 & $12-374$ \\
\hline \multirow[t]{2}{*}{ Number of surgeries } & 2.6 & $1-9$ & 2.5 & $1-9$ \\
\hline & $\mathrm{n}$ & $\%$ & $\mathrm{n}$ & $\%$ \\
\hline \multicolumn{5}{|l|}{ Gender } \\
\hline Female & 230 & 85 & 56 & 78 \\
\hline Male & 42 & 15 & 16 & 22 \\
\hline \multicolumn{5}{|l|}{ Localization } \\
\hline Knee & 199 & 73 & 64 & 89 \\
\hline Hip & 25 & 9 & 8 & 11 \\
\hline Ankle & 31 & 11.5 & - & - \\
\hline Elbow & 8 & 3 & - & - \\
\hline Foot & 4 & 1.5 & - & - \\
\hline Shoulder & 4 & 1.5 & - & - \\
\hline Hand & 1 & 0.5 & - & - \\
\hline \multicolumn{5}{|l|}{ Type of disease } \\
\hline Diffuse disease & $\mathrm{U}$ & $\mathrm{U}$ & 58 & 81 \\
\hline Localized disease & $\mathrm{U}$ & $\mathrm{U}$ & 14 & 19 \\
\hline \multicolumn{5}{|l|}{ Pre-operative complaints } \\
\hline Pain & 202 & 74 & 25 & 35 \\
\hline -At rest & $\mathrm{U}$ & $\mathrm{U}$ & 19 & 26 \\
\hline -During exercise & $\mathrm{U}$ & $\mathrm{U}$ & 19 & 26 \\
\hline -At night & $\mathrm{U}$ & $\mathrm{U}$ & 16 & 22 \\
\hline Swelling & 202 & 74 & 25 & 35 \\
\hline Stiffness/limited ROM & 113 & 41 & 25 & 35 \\
\hline Locking & 61 & 22 & $\mathrm{U}$ & $\mathrm{U}$ \\
\hline \multicolumn{5}{|l|}{ Surgical treatment } \\
\hline Arthroscopic synovectomy & 118 & 43 & 38 & 53 \\
\hline Open synovectomy & 97 & 36 & 34 & 47 \\
\hline Combined hybrid synovectomy & 10 & 4 & - & - \\
\hline Unknown & 47 & 17 & - & - \\
\hline
\end{tabular}

$U$ unknown, $R O M$ range of motion
Table 3 Factors of influence on recurrence rate after synovectomy for PVNS in the knee or hip

\begin{tabular}{llll}
\hline & OR & $95 \%$ CI & $p$-value \\
\hline Diffuse disease & 16 & $3.2-85$ & 0.001 \\
Arthroscopic synovectomy & 2.2 & $0.74-6.6$ & 0.16 \\
Localization in knee & 1.3 & $0.27-5.9$ & 0.77 \\
Arthroscopic synovectomy & 1.7 & $0.67-4.5$ & 0.26 \\
\hline
\end{tabular}

$O R$ odds ratio, $C I$ confidence interval

tency checks between both surveys. Primary surgery was arthroscopic synovectomy $(n=38)$ or open synovectomy $(n=34)$. Recurrence rates are listed in Table 2. Mean number of surgeries was 2.5 (1-9). Recurrences were treated with open synovectomy $(n=16)$, arthroscopic synovectomy $(n=15)$ or complete resection and joint arthroplasty $(n=12)$. Total hip arthroplasty was required in $6 / 8$ and total knee arthroplasty in $7 / 64(p=0.004)$. Adjuvant treatment was radiation therapy $(n=12)$, ${ }^{90}$ Yttrium $(n=9)$, imatinib $(n=1)$ or nilotinib $(n=1)$. Diffuse disease increased recurrence risk (odds ratio $[\mathrm{OR}]=16 ; 95 \%$ confidence interval $[\mathrm{CI}]=3.2-85$; $p=0.001$; Table 3). Mean functional and QOL results did not differ significantly after primary arthroscopic or open synovectomy (Table 4). Final surgery resulted in a mean TESS of 88 after arthroscopic synovectomy, 68 after open synovectomy and 69 after total joint arthroplasty $(p=0.017)$. Joint replacement surgery resulted in lower functional scores compared with joint salvage: TESS 69 vs. $82(p=0.010)$, KOOS 34 vs. 55 $(p=0.031)$ and SF-36 49 vs. $66(p=0.020)$. Mean ROM was lower for patients with diffuse disease (117 vs. 151 degrees; $p=0.024$ ) and for recurrent disease requiring repeat surgery (113 vs. 138 degrees; $p=0.046$ ).

Consistency checks were performed with Cohen's kappa statistic for intra-rater agreement; 66 patients could be crosslinked between both surveys through IP address. Cohen's kap-
Table 2 Local recurrences after synovectomy for PVNS in the knee or hip

\begin{tabular}{|c|c|c|c|c|c|c|c|}
\hline \multirow[t]{2}{*}{ Group $1(n=272)$} & \multicolumn{2}{|c|}{$\begin{array}{l}\text { Arthroscopic } \\
\text { synovectomy }\end{array}$} & \multicolumn{2}{|c|}{$\begin{array}{l}\text { Open } \\
\text { synovectomy }\end{array}$} & \multicolumn{2}{|c|}{$\begin{array}{l}\text { Combined } \\
\text { synovectomy* }\end{array}$} & \multirow[t]{2}{*}{$p$-value } \\
\hline & $\%$ & $\mathrm{n}$ & $\%$ & $\mathrm{n}$ & $\%$ & $\mathrm{n}$ & \\
\hline Total group & $58 \%$ & $69 / 118$ & $36 \%$ & $35 / 97$ & $50 \%$ & $5 / 10$ & 0.003 \\
\hline \multicolumn{8}{|l|}{ Group $2(n=72)$} \\
\hline Total group & $67 \%$ & $26 / 39$ & $51 \%$ & $17 / 33$ & - & - & 0.19 \\
\hline Localized disease & $25 \%$ & $2 / 8$ & $0 \%$ & $0 / 6$ & - & - & 0.31 \\
\hline Diffuse disease & $77 \%$ & $23 / 30$ & $64 \%$ & $18 / 28$ & - & - & 0.23 \\
\hline
\end{tabular}

*Combined arthroscopic and open synovectomy 
Table 4 Functional outcome and QOL after primary synovectomy for PVNS in the knee or hip

\begin{tabular}{|c|c|c|c|c|c|}
\hline & \multicolumn{2}{|c|}{ Arthroscopic synovectomy } & \multicolumn{2}{|c|}{ Open synovectomy } & \multirow[t]{2}{*}{$p$-value } \\
\hline & mean & range & mean & range & \\
\hline ROM (degrees) & 124 & $80-170$ & 129 & $65-170$ & 0.65 \\
\hline KOOS & 49 & $8-92$ & 58 & $34-92$ & 0.24 \\
\hline HOOS & 62 & $51-72$ & 53 & $31-67$ & 0.56 \\
\hline TESS & 78 & $33-100$ & 82 & $63-97$ & 0.86 \\
\hline SF-36 & 61 & $11-100$ & 66 & $21-98$ & 0.41 \\
\hline
\end{tabular}

ROM range of motion, TESS Toronto extremity salvage score, KOOS knee injury and osteoarthritis outcome score, HOOS hip disability and osteoarthritis outcome score, $S F-36$ short form 36 health survey pa statistic for the two online surveys was outstanding for tumour localization with $\kappa=0.95(p<0.001)$, joint arthroplasty with $\kappa=0.88(p<0.001)$ and adjuvant treatment with $\kappa=0.82(p<0.001)$; and substantial for primary surgical treatment with $\kappa=0.78(p<0.001)$ and recurrences with $\kappa=0.68(p<0.001)$.

\section{Discussion}

In this study, we investigated whether we could use crowdsourcing via Facebook and online surveys for medical research purposes on PVNS. We set out to correlate functional outcome and QOL to patient characteristics, surgical procedures and oncological outcome in patients with PVNS who were enrolled in a patient community on Facebook. We concluded that the recurrence risk was highest for diffuse disease and after arthroscopic synovectomy. Lower functional results were reported for patients with diffuse disease, for patients with recurrences requiring repeat surgery, and for patients eventually requiring joint replacement surgery.

To determine whether the results of this study are reliable and whether crowdsourcing via social media is feasible in medical research, we performed consistency tests by evaluating intra-rater agreement for patients' answers between both online surveys, which was substantial to outstanding for most study variables. The somewhat lower agreement in follow-up questions can be explained by the time interval between the two surveys as patients may have experienced a recurrence after filling out the first survey. In addition, we compared our results with previous publications on PVNS to assess the representativeness of our sampling frame [17] (Tables 3 and 5). An advantage of this comparison may be the determination of accurateness of the use of crowdsourcing and the reliability of the obtained data. Possible disadvantages of crowdsourcing may include selection bias through the sampling method, inhibiting comparison of results with previously published reports. The average age of 32 years at onset of disease in this study matches known epidemiology of PVNS ( $<40$ years) $[1,2,36]$. Localization of PVNS in this study (73\% knee, $9 \%$ hip, $18 \%$ other) was also comparable to percentages described in literature (75\% knee, $15 \%$ hip, $10 \%$ other). Multifocal PVNS is rare and was not reported in this study [36]. Women were over-represented $(78 \%)$ when compared with known epidemiology of PVNS; possibly indicating that women are more likely to be on Facebook and to seek information via support groups [37]. All study variables were distributed equally between men and women. Women scored somewhat lower on SF-36 subdomains physical functioning, pain and PCS; all other functional and QOL scores did not differ between men and women (results not shown). Proportions of arthroscopic and open synovectomy [4] and total knee or hip arthroplasty were comparable to those previously published $[38,39]$.

Mean follow-up of approximately six years (range 131) was longer when compared with the majority of previously published reports [3, 19, 23, 29, 31, 32]. Together with the relatively high recurrence rates in this study (58-77\% after arthroscopic and 36-64\% after open synovectomy), this may indicate that to date, an underestimate of the true local recurrence rate of PVNS has been published.

Functional results after surgical treatment for PVNS have been reported (Table 5) [3, 18-32], but comparison is difficult, as functional results were not specified for diffuse and localized disease, type of surgery, primary or recurrent disease and different localizations. In this study, there was no difference in functional outcome and QOL between patients who underwent primary arthroscopic or open synovectomy. However, the long course of disease and the need for multiple surgeries has previously been reported to result in worse functional results in a large number of patients [23]; and in this study, lower functional results were also reported for patients with diffuse disease, with recurrences requiring repeat surgery, 
Table 5 Literature overview on oncological and functional results after arthroscopic and open synovectomy for PVNS

\begin{tabular}{|c|c|c|c|c|c|c|c|c|c|c|}
\hline \multirow[t]{2}{*}{ Study } & \multirow[t]{2}{*}{$\mathrm{n}$} & \multirow[t]{2}{*}{ Sex } & \multirow{2}{*}{$\begin{array}{l}\text { Age } \\
\text { (years) } \\
\text { mean } \\
\text { (range) }\end{array}$} & \multirow{2}{*}{$\begin{array}{l}\text { Follow-up } \\
\text { (months) } \\
\text { mean } \\
\text { (range) }\end{array}$} & \multirow[t]{2}{*}{ Localization } & \multirow[t]{2}{*}{ PVNS type } & \multirow[t]{2}{*}{ Surgery } & \multirow{2}{*}{$\begin{array}{l}\text { Recurrence } \\
\text { rate } \\
\%\end{array}$} & \multicolumn{2}{|c|}{ Functional outcome } \\
\hline & & & & & & & & & & mean (range) \\
\hline $\begin{array}{l}\text { De Visser } \\
\quad \text { et al. [18] }\end{array}$ & 38 & $\begin{array}{l}\text { F18, } \\
\text { M20 }\end{array}$ & $32(12-72)$ & $48(12-228)$ & knee, hip, ankle & $\begin{array}{l}29 \text { diffuse } \\
9 \text { localized }\end{array}$ & unspecified & unspecified & MSTS & $24(15-30)$ \\
\hline $\begin{array}{l}\text { Zvijac et al. } \\
\qquad[19]\end{array}$ & 14 & F7, M7 & $35(19-64)$ & $42(8-83)$ & knee & $\begin{array}{l}12 \text { diffuse } \\
2 \text { localized }\end{array}$ & $\begin{array}{l}\text { arthroscopic } \\
\text { synovectomy }\end{array}$ & $\begin{array}{l}\text { diffuse } 14 \% \\
\text { localized } 0 \%\end{array}$ & other & $\begin{array}{l}10 \text { excellent/good, } 2 \text { fair, }^{1} \\
2 \text { poor }^{1}\end{array}$ \\
\hline $\begin{array}{l}\text { Shabat et al. } \\
{[20]}\end{array}$ & 10 & F2, M8 & NR (15-49) & $72(30-144)$ & knee, ankle, hip & diffuse & unspecified & $10 \%$ & MSTS & 9 excellent, 1 unknown ${ }^{1}$ \\
\hline $\begin{array}{l}\text { Chin et al. } \\
\quad[21]\end{array}$ & 40 & $\begin{array}{l}\text { F17, } \\
\text { M23 }\end{array}$ & $35(14-68)$ & $60(18-96)$ & knee & diffuse & $\begin{array}{l}\text { open } \\
\text { synovectomy }\end{array}$ & $17 \%$ & $\begin{array}{l}\text { KSS } \\
\text { other }^{2}\end{array}$ & $\begin{array}{l}92(55-100) \\
92(0-100)\end{array}$ \\
\hline $\begin{array}{l}\text { De Ponti et al. } \\
\quad[22]\end{array}$ & 19 & F10, M9 & $59(37-83)$ & $60(12-128)$ & knee & $\begin{array}{l}15 \text { diffuse } \\
4 \text { localized }\end{array}$ & $\begin{array}{l}\text { arthroscopic } \\
\text { synovectomy }\end{array}$ & $\begin{array}{l}\text { diffuse } 50 \% \\
\text { localized } 0 \%\end{array}$ & other ${ }^{3}$ & $\begin{array}{l}\text { Complete arthroscopy: } \\
\text { excellent }{ }^{1} \\
\text { Partial arthroscopy: } \\
\quad \text { good }^{1}\end{array}$ \\
\hline $\begin{array}{l}\text { Chiari et al. } \\
\text { [23] }\end{array}$ & 42 & $\begin{array}{l}\text { F27, } \\
\text { M15 }\end{array}$ & $40(6-76)$ & $80(26-194)$ & $\begin{array}{l}\text { knee, ankle, hip, } \\
\text { foot, } \\
\text { shoulder, } \\
\text { hand }\end{array}$ & $\begin{array}{l}19 \text { diffuse } \\
23 \text { localized }\end{array}$ & $\begin{array}{l}\text { open } \\
\text { synovectomy }\end{array}$ & overall $24 \%$ & MSTS & $28(18-30)$ \\
\hline Wu et al. [24] & 9 & F4, M5 & $31(19-51)$ & $67(37-103)$ & knee & diffuse & $\begin{array}{l}\text { open } \\
\text { synovectomy }\end{array}$ & $11 \%$ & $\begin{array}{l}\text { KSS-knee } \\
\text { KSS- } \\
\quad \text { function }\end{array}$ & $\begin{array}{l}94(86-98) \\
97(80-100)\end{array}$ \\
\hline $\begin{array}{l}\text { Dines et al. } \\
\quad[25]\end{array}$ & 26 & $\begin{array}{l}\text { F11, } \\
\text { M15 }\end{array}$ & $36(12-68)$ & $66(46-123)$ & knee & localized & $\begin{array}{l}14 \text { open } \\
\text { synovectomy } \\
12 \text { arthroscopic } \\
\text { synovectomy }\end{array}$ & $0 \%$ & $\mathrm{LKS}^{4}$ & $95(71-100)$ \\
\hline $\begin{array}{l}\text { Ozturk et al. } \\
\quad[26]\end{array}$ & 7 & $\mathrm{~F} 4, \mathrm{M} 3$ & $45(20-68)$ & $48(24-97)$ & knee & diffuse & $\begin{array}{l}4 \text { arthroscopic } \\
\text { synovectomy } \\
3 \text { open } \\
\text { synovectomy }\end{array}$ & $0 \%$ & MSTS & $21(12-26)$ \\
\hline $\begin{array}{l}\text { Nassar et al. } \\
{[27]}\end{array}$ & 12 & F4, M8 & NR (19-49) & $27(20-36)$ & knee & diffuse & $\begin{array}{l}\text { open } \\
\text { synovectomy }\end{array}$ & $0 \%$ & MSTS & $25.5(24-27)$ \\
\hline Liu et al. [28] & 22 & F14, M8 & $24(16-35)$ & $22(18-28)$ & knee & localized & $\begin{array}{l}\text { arthroscopic } \\
\text { synovectomy }\end{array}$ & $14 \%$ & $\begin{array}{l}\text { LKS } \\
\text { IKDC }\end{array}$ & $\begin{array}{l}95(\mathrm{SD} 3.5) \\
93(\mathrm{SD} 2.4)\end{array}$ \\
\hline $\begin{array}{l}\text { Akinci et al. } \\
\text { [29] }\end{array}$ & 19 & F10, M9 & $43(\mathrm{NR})$ & $80(15-156)$ & knee & $\begin{array}{l}15 \text { diffuse } \\
4 \text { localized }\end{array}$ & $\begin{array}{l}\text { open } \\
\text { synovectomy }\end{array}$ & overall $26 \%$ & KSS & 8 perfect, 9 good, 2 bad $^{1}$ \\
\hline $\begin{array}{l}\text { Griffin et al. } \\
\quad[30]\end{array}$ & 50 & $\begin{array}{l}\text { F30, } \\
\text { M20 }\end{array}$ & $38(18-74)$ & $94(19-330)$ & $\begin{array}{l}\text { knee, ankle, hip, } \\
\text { foot, } \\
\text { hand, wrist }\end{array}$ & diffuse & unspecified & $6 \%$ & $\begin{array}{l}\text { MSTS- } 87^{5} \\
\text { MSTS- }^{5} 3^{5} \\
\text { TESS }^{5} \\
\text { other }^{5}\end{array}$ & $\begin{array}{l}31(25-35) \\
28(19-30) \\
90(65-99) \\
7 \text { excellent, } 34 \text { good, } 5 \\
\quad \text { fair, } 4 \text { poor }{ }^{1}\end{array}$ \\
\hline $\begin{array}{l}\text { Nakahara } \\
\quad \text { et al. [31] }\end{array}$ & 17 & $\mathrm{~F} 7, \mathrm{M} 10$ & $33(\mathrm{SD} 17.2)$ & $65(10-146)$ & knee & diffuse & $\begin{array}{l}\text { open } \\
\text { synovectomy }\end{array}$ & $12 \%$ & KSS & $97(76-100)$ \\
\hline Chen et al. [3] & 19 & F10, M9 & $43(29-59)$ & $98(42-143)$ & knee & diffuse & $\begin{array}{l}\text { open } \\
\text { synovectomy }\end{array}$ & $11 \%$ & TLKS & $93(86-100)$ \\
\hline $\begin{array}{l}\text { Loriaut et al. } \\
\qquad[32]\end{array}$ & 30 & NR & $46(23-71)$ & $75(12-144)$ & knee & localized & $\begin{array}{l}\text { arthroscopic } \\
\text { synovectomy }\end{array}$ & $20 \%$ & LKS & $86(83-88)$ \\
\hline $\begin{array}{l}\text { Van der } \\
\text { Heijden } \\
\text { et al. [33] }\end{array}$ & 30 & $\begin{array}{l}\text { F17, } \\
\text { M13 }\end{array}$ & $34(6-73)$ & $95(24-403)$ & knee & diffuse & $\begin{array}{l}14 \text { open } \\
\text { synovectomy } \\
16 \text { arthroscopic } \\
\text { synovectomy }\end{array}$ & $\begin{array}{c}\text { open } 28 \% \\
\text { arthroscopy } \\
94 \%\end{array}$ & $\begin{array}{l}\text { KOOS } \\
\text { MSTS } \\
\text { TESS } \\
\text { SF-36 }\end{array}$ & $\begin{array}{l}59(12-99) \\
21(8-30) \\
80(45-100) \\
70(26-98)\end{array}$ \\
\hline Jain et al. [34] & 40 & F9, M31 & $44(21-76)$ & $84(24-120)$ & knee & $\begin{array}{l}29 \text { diffuse } \\
11 \text { localized }\end{array}$ & $\begin{array}{l}\text { arthroscopic } \\
\text { synovectomy }\end{array}$ & $\begin{array}{l}41 \% \\
0 \%\end{array}$ & LKS & 31 excellent, 8 good \\
\hline Ma et al. [35] & 75 & $\begin{array}{l}\text { F48, } \\
\text { M27 }\end{array}$ & $46(15-80)$ & 41 & knee, hip, ankle & $\begin{array}{l}67 \text { diffuse } \\
8 \text { localized }\end{array}$ & $\begin{array}{l}\text { open or } \\
\text { arthroscopic } \\
\text { synovectomy } \\
\text { with or } \\
\text { without } \\
\text { artroplasty }\end{array}$ & $16 \%$ & NR & \\
\hline Current study & 272 & $\begin{array}{l}\text { F230, } \\
\text { M42 }\end{array}$ & 32 (SD 12) & NR & $\begin{array}{l}\text { knee, hip, ankle, } \\
\text { elbow, foot, } \\
\text { shoulder, } \\
\text { hand }\end{array}$ & $\begin{array}{l}\text { diffuse and } \\
\text { localized }\end{array}$ & $\begin{array}{l}97 \text { open } \\
\text { synovectomy } \\
118 \text { arthroscopic } \\
\text { synovectomy }\end{array}$ & $\begin{array}{c}\text { open } 36 \% \\
\text { arthroscopy } \\
58 \% \\
\text { hybrid } 50 \%\end{array}$ & NR & \\
\hline
\end{tabular}


Table 5 (continued)

\begin{tabular}{|c|c|c|c|c|c|c|c|c|c|c|}
\hline \multirow[t]{2}{*}{ Study } & \multirow[t]{2}{*}{$\mathrm{n}$} & \multirow[t]{2}{*}{ Sex } & \multirow{2}{*}{$\begin{array}{l}\text { Age } \\
\text { (years) } \\
\text { mean } \\
\text { (range) }\end{array}$} & \multirow{2}{*}{$\begin{array}{l}\text { Follow-up } \\
\text { (months) } \\
\text { mean } \\
\text { (range) }\end{array}$} & \multirow[t]{2}{*}{ Localization } & \multirow[t]{2}{*}{ PVNS type } & \multirow[t]{2}{*}{ Surgery } & \multirow{2}{*}{$\begin{array}{l}\text { Recurrence } \\
\text { rate } \\
\%\end{array}$} & \multicolumn{2}{|c|}{ Functional outcome } \\
\hline & & & & & & & & & & mean (range) \\
\hline & & & & & & & $\begin{array}{l}10 \text { combined } \\
\text { open and } \\
\text { arthroscopic } \\
\text { synovectomy } \\
47 \text { unknown }\end{array}$ & & & \\
\hline & 72 & $\begin{array}{l}\text { F56, } \\
\text { M16 }\end{array}$ & 31 (SD 12) & $70(12-374)$ & knee, hip & $\begin{array}{l}58 \text { diffuse } \\
14 \text { localized }\end{array}$ & $\begin{array}{l}34 \text { open } \\
\text { synovectomy } \\
38 \text { arthroscopic } \\
\text { synovectomy }\end{array}$ & $\begin{array}{c}\text { open } 51 \% \\
\text { arthroscopy } \\
67 \%\end{array}$ & $\begin{array}{l}\text { KOOS } \\
\text { HOOS } \\
\text { TESS } \\
\text { SF-36 }\end{array}$ & $\begin{array}{l}58(34-92) / 49(8-92)^{6} \\
53(31-67) / 62(51-72)^{6} \\
82(63-97) / 78(33-100)^{6} \\
66(21-98) / 61(11-100)^{6}\end{array}$ \\
\hline
\end{tabular}

$F$ female, $M$ male, $N R$ not reported, KSS Knee Society score, TLKS Tegner-Lysholm knee score, $L K S$ Lysholm knee scale, IKDC International Knee Documentation Committee, $S D$ standard deviation

${ }^{1}$ Functional outcome was not further specified

${ }^{2}$ Functional outcome was based on pain, walking status, joint swelling, effusion, crepitus, locking, instability and ROM

${ }^{3}$ Functional outcome was based on pain, synovitis, joint swelling and ROM

${ }^{4}$ Functional outcome was obtained from $10 / 26$ patients

${ }^{5}$ Functional outcome was obtained from $14 / 50$ patients

${ }^{6}$ Functional results after open synovectomy/arthroscopic synovectomy

and for patients eventually requiring joint replacement surgery.

Our study has several limitations. First, the low participation rate in this study $(26.3 \%)$ may be explained by several arguments, including the voluntary character of the survey, and the attention paid to the survey by the Facebook members; maybe they didn't see the requests on their wall, didn't have time to compile the questionnaires or didn't feel the need to add to a scientific research project. This is also of concern when similar studies involving social media are performed in medicine, as it may induce selection bias. In regards to this potential selection bias, patients who are currently unsatisfied with their situation may be more likely to enrol in a patient community on Facebook and to complete online surveys concerning functional and QOL results. However, the opposite may also be true for satisfied patients who are willing to share their experience and to improve awareness on this rare disease. Second, patients were not uniformly diagnosed and treated for PVNS, as they were collected from 23 different countries and treated by various surgeons without distinguishing between peripheral and tertiary referral centres. However, we believe that it represents a randomly chosen group of patients, perfectly reflecting the current worldwide situation and underlining the importance of centralization of care for musculoskeletal tumours [40]. Third, the accuracy of surgical data is subjective to the understanding of patients; data obtained from patients were not verified by contacting treating physicians. We considered this beyond the scope of the current study but we deem it valuable in future crowdsourcing studies via social media. Yet, PROMs are considered more reliable in reporting subjective patient outcomes when compared to the evaluation by treating physician, and are more often required by the FDA $[8,41]$.

In addition to using social media for crowdsourcing purposes in rare diseases, it may also increase the readership and impact of scientific publications apart from the indexed impact factor.

An increasing number of scientific papers can be found through social media such as Facebook, Twitter, LinkedIn and ResearchGate; and journals should explore these platforms and use it in a constructive way to increase the scientific exposure [42].

In conclusion, gathering data via crowdsourcing in a patient community on Facebook seems a promising and innovative way of evaluating rare diseases, as it provides for a representative and large sample of patients with long-term follow-up and valid clinical outcome data. The results of this study suggest that local recurrence risk and functional outcome were both negatively influenced by diffuse disease, which comprises a large part of the joint, is difficult to resect completely and often requires repeat surgery, especially after arthroscopic synovectomy.

Acknowledgments The authors would like to thank all the patients of the PVNS patient community on Facebook for compiling the questionnaires.

\section{Compliance with ethical standards}

Conflict of interest The authors declare that they have no conflict of interest. 


\section{Appendix: Data reporting guidelines}

\section{Appendix 1: CHERRIES checklist}

Table 6 Checklist for reporting results of internet E-surveys (CHERRIES)

\begin{tabular}{|c|c|c|}
\hline Item category & Checklist item & This study \\
\hline Design & Describe survey design & $\begin{array}{l}\text { Target population is } 1112 \text { patients enrolled in "PVNS is pants!!" community } \\
\text { on Facebook }\end{array}$ \\
\hline \multirow[t]{3}{*}{$\begin{array}{l}\text { IRB approval and informed consent } \\
\text { process }\end{array}$} & IRB approval & $\begin{array}{l}\text { Approval of the ethics committee of our institution was waived because this } \\
\text { study did not fall under the scope of the Dutch law on Medical Research } \\
\text { Involving Human Subjects Act (WMO). }\end{array}$ \\
\hline & Informed consent & $\begin{array}{l}\text { Informed consent was included in the questionnaires. Length of survey was } \\
\text { explained on the first page of survey, investigators } \mathrm{PhD} \text { student and } \\
\text { consultant orthopedic oncologist from a tertiary centre in the Netherlands, } \\
\text { purpose of study to increase knowledge and awareness on this rare disease } \\
\text { including PROMs. }\end{array}$ \\
\hline & Data protection & Results from survey visible to researchers only, password protected. \\
\hline Development and pre-testing & Development and testing & $\begin{array}{l}\text { Survey developed via surveymonkey.com, usability and technical } \\
\text { functionality was tested by researchers. }\end{array}$ \\
\hline \multirow{3}{*}{$\begin{array}{l}\text { Recruitment process and description } \\
\text { of the sample having access to the } \\
\text { questionnaire }\end{array}$} & $\begin{array}{l}\text { Open survey versus closed } \\
\text { survey }\end{array}$ & Open survey on community on Facebook \\
\hline & Contact mode & $\begin{array}{l}\text { Contact with participants was made through the Facebook community, links } \\
\text { to the surveys were provided here. }\end{array}$ \\
\hline & Advertising the survey & $\begin{array}{l}\text { The e-survey was announced on the Facebook community wall with a first } \\
\text { notice of the study, and repeated requests after 1, 2, 5, } 6 \text { and } 10 \text { months. } \\
\text { See Appendix I (below). }\end{array}$ \\
\hline \multirow[t]{10}{*}{ Survey administration } & Web/E-mail & $\begin{array}{l}\text { E-survey posted on a website (i.e. Facebook community on PVNS). } \\
\text { Responses were automatically captured into an SPSS } 20.0 \text { file, which was } \\
\text { only accessible to the researchers. }\end{array}$ \\
\hline & Context & $\begin{array}{l}\text { Facebook community on PVNS, mostly patients but also family members and } \\
\text { the research from this study. Normally looking to share information and } \\
\text { personal experiences concerning this rare disease, its (surgical) treatment, } \\
\text { recovery duration, tips and tricks; so best summarized as an information } \\
\text { and support group. } \\
\text { Women are somewhat overrepresented when compared with known } \\
\text { epidemiology of PVNS, and may be more likely to seek information via } \\
\text { support groups? } \\
\text { Patients from countries all over the world, including developing and } \\
\text { developed countries, age groups of Facebook users matches known } \\
\text { epidemiology. }\end{array}$ \\
\hline & Mandatory/voluntary & Voluntary survey \\
\hline & Incentives & No incentives were offered \\
\hline & Time/date & $\begin{array}{l}\text { December 2012-April } 2014 \\
\text { May 2013-May } 2014\end{array}$ \\
\hline & $\begin{array}{l}\text { Randomization of items or } \\
\text { questionnaires }\end{array}$ & No randomization, standardizes PROMs \\
\hline & Adaptive questioning & No adaptive questioning, standardized PROMs \\
\hline & Number of items & $\begin{array}{l}\text { General survey }=20 \\
\text { SF36 }=36 \\
\text { KOOS }=42 \\
\text { HOOS }=40 \\
\text { TESS }=30\end{array}$ \\
\hline & Number of screens (pages) & 1 webpage per survey, if applicable \\
\hline & Completeness check & $\begin{array}{l}\text { Yes, completeness checks were done through JAVAScript, consistency checks } \\
\text { were not performed, not applicable options were included, general } \\
\text { questions included some open and some multiple option questions, } \\
\text { PROMs were standardized validated questionnaires with enforced one- } \\
\text { response-option. }\end{array}$ \\
\hline
\end{tabular}


Table 6 (continued)

\begin{tabular}{|c|c|c|}
\hline Item category & Checklist item & This study \\
\hline \multirow{5}{*}{ Response rates } & Review step & Respondents were able to review and change their answers \\
\hline & Unique site visitor & Unique site visitors were determined by IP addresses. \\
\hline & $\begin{array}{l}\text { View rate (ratio of unique } \\
\text { survey visitors/unique site } \\
\text { visitors) }\end{array}$ & $\begin{array}{l}\text { View rate } \\
\text { Total } 274 / 1112=24,6 \% \\
\text { SF36 } 131 / 1112=11.8 \% \\
\text { KOOS } 59 / 1112=5.3 \% \\
\text { HOOS } 9 / 1112=0.8 \% \\
\text { TESS } 34 / 1112=3.1 \%\end{array}$ \\
\hline & $\begin{array}{l}\text { Participation rate (ratio of } \\
\text { unique visitors who agreed to } \\
\text { participate/unique first } \\
\text { survey page visitors) }\end{array}$ & $\begin{array}{l}\text { Participation rate } \\
\text { Total } 270 / 274=98.5 \% \\
\text { SF36 } 131 / 131=100 \% \\
\text { KOOS } 59 / 59=100 \% \\
\text { HOOS } 9 / 9=100 \% \\
\text { TESS } 34 / 34=100 \%\end{array}$ \\
\hline & $\begin{array}{l}\text { Completion rate (ratio of users } \\
\text { who finished the survey/users } \\
\text { who agreed to participate) }\end{array}$ & $\begin{array}{l}\text { Completion/completeness rate } \\
\text { SF36 } 111 / 131=84.7 \% \\
\text { KOOS } 51 / 59=86.4 \% \\
\text { HOOS } 9 / 9=100 \% \\
\text { TESS } 34 / 34=100 \%\end{array}$ \\
\hline \multirow{4}{*}{$\begin{array}{l}\text { Preventing multiple entries from the } \\
\text { same individual }\end{array}$} & Cookies used & No cookies were used \\
\hline & IP check & $\begin{array}{l}\text { Duplicate database entries having the same user ID/IP address were } \\
\text { eliminated before analysis; the most recent entries were included. }\end{array}$ \\
\hline & Log file analysis & See above \\
\hline & Registration & N. A. \\
\hline \multirow[t]{3}{*}{ Analysis } & $\begin{array}{l}\text { Handling of incomplete } \\
\text { questionnaires }\end{array}$ & Only completed questionnaires were analyzed. \\
\hline & $\begin{array}{l}\text { Questionnaires submitted with } \\
\text { an atypical timestamp }\end{array}$ & N.A. \\
\hline & Statistical correction & $\begin{array}{l}\text { Propensity scores to adjust for the non-representative sample were not used in } \\
\text { this study }\end{array}$ \\
\hline
\end{tabular}

Appendix I: Survey announcements 12-05-2013

Announcement of study initiative: Knowledge on QOL and functional results after treatment of PVNS is lacking; validated questionnaires will be launched here soon and your valuable data will be used to create awareness and improve treatment protocols as were recently published [REF JSO, JBJS Br].

01-08-2013 Announcement including link to survey: Please fill in this first Facebook-based research initiative on functional outcome and quality of life of patients with PVNS and allow us to evaluate your quality of life and limb or joint function in order to improve the current treatment protocols and therewith the functional outcome and quality of life of patients with PVNS in the future. All data will be handled with care and are only used according to the rules of good clinical and research practice. Data stored will not be traceable to your personal details, all will be anonymized.

Further announcements including link to survey were provided after 1, 2, 5, 6 and 10 months. (19-08-2013, 31-08-2013, 03-09-2013, 1709-2013, 22-09-2013, 22-12-2013, 31-12-2013, 29-01-2014, 23-05-2014).

Open Access This article is distributed under the terms of the Creative Commons Attribution 4.0 International License (http:// creativecommons.org/licenses/by/4.0/), which permits unrestricted use, distribution, and reproduction in any medium, provided you give appropriate credit to the original author(s) and the source, provide a link to the Creative Commons license, and indicate if changes were made.

\section{References}

1. de St. Aubain SN, van de Rijn M (2013) Tenosynovial giant cell tumour, diffuse type. In: Fletcher CD, Bridge JA, Hogendoorn PC, Mertens F (eds) WHO classification of tumours of soft tissue and bone, 4th edn. IARC Press, Lyon, pp 102-103

2. de St. Aubain SN, van de Rijn M (2013) Tenosynovial giant cell tumour, localized type. In: Fletcher CD, Bridge JA, Hogendoorn PC, Mertens F (eds) WHO classification of tumours of soft tissue and bone. IARC Press, Lyon, pp 100-101

3. Chen WM, Wu PK, Liu CL (2012) Simultaneous anterior and posterior synovectomies for treating diffuse pigmented villonodular synovitis. Clin Orthop Relat Res 470:1755-1762

4. Van der Heijden L, Gibbons CL, Hassan AB, Kroep JR, Gelderblom H et al (2013) A multidisciplinary approach to giant cell tumors of tendon sheath and synovium-a critical appraisal of literature and treatment proposal. J Surg Oncol 107:433-445

5. Van der Heijden L, Gibbons CL, Dijkstra PD, Kroep JR, van Rijswijk CS et al (2012) The management of diffuse-type giant cell tumour (pigmented villonodular synovitis) and giant cell tumour of tendon sheath (nodular tenosynovitis). J Bone Joint Surg (Br) 94:882-888

6. Wicks P (2014) Could digital patient communities be the launch pad for patient-centric trial design? Trials 15:172 
7. Wicks P, Vaughan T, Heywood J (2014) Subjects no more: what happens when trial participants realize they hold the power? BMJ 348:g368

8. Gnanasakthy A, Mordin M, Clark M, DeMuro C, Fehnel S et al (2012) A review of patient-reported outcome labels in the United States: 2006 to 2010. Value Health 15:437-442

9. de Groot IB, Favejee MM, Reijman M, Verhaar JA, Terwee CB (2008) The Dutch version of the knee injury and osteoarthritis outcome score: a validation study. Health Qual Life Outcomes 6:16

10. Paradowski PT, Bergman S, Sunden-Lundius A, Lohmander LS, Roos EM (2006) Knee complaints vary with age and gender in the adult population. Population-based reference data for the knee injury and osteoarthritis outcome score (KOOS). BMC Musculoskelet Disord 7

11. de Groot IB, Reijman M, Terwee CB, Bierma-Zeinstra S, Favejee MM et al (2009) Validation of the Dutch version of the Hip disability and osteoarthritis outcome score. Osteoarthr Cartil 17:132

12. Davis AM, Wright JG, Williams JI, Bombardier C, Griffin A et al (1996) Development of a measure of physical function for patients with bone and soft tissue sarcoma. Qual Life Res 5:508-516

13. Aaronson NK, Muller M, Cohen PD, Essink-Bot ML, Fekkes M et al (1998) Translation, validation, and norming of the Dutch language version of the SF-36 health survey in community and chronic disease populations. J Clin Epidemiol 51:1055-1068

14. Eysenbach $G$ (2004) Improving the quality of web surveys: the checklist for reporting results of internet E-surveys (CHERRIES). J Med Internet Res 6:e34

15. Kelley K, Clark B, Brown V, Sitzia J (2003) Good practice in the conduct and reporting of survey research. Int J Qual Health Care 15: 261-266

16. Landis JR, Koch GG (1977) The measurement of observer agreement for categorical data. Biometrics 33:159-174

17. Bhutta CB (2012) Not by the book-facebook as a sampling frame. Sociol Methods Res 41:57-88

18. de Visser E, Veth RP, Pruszczynski M, Wobbes T, van de Putte LB (1999) Diffuse and localized pigmented villonodular synovitis: evaluation of treatment of 38 patients. Arch Orthop Trauma Surg 119:401-404

19. Zvijac JE, Lau AC, Hechtman KS, Uribe JW, Tjin ATE (1999) Arthroscopic treatment of pigmented villonodular synovitis of the knee. Arthroscopy 15:613-617

20. Shabat S, Kollender Y, Merimsky O, Isakov J, Flusser G et al (2002) The use of surgery and yttrium 90 in the management of extensive and diffuse pigmented villonodular synovitis of large joints. Rheumatology (Oxford) 41:1113-1118

21. Chin KR, Barr SJ, Winalski C, Zurakowski D, Brick GW (2002) Treatment of advanced primary and recurrent diffuse pigmented villonodular synovitis of the knee. J Bone Joint Surg Am 84-A: 2192-2202

22. De Ponti A, Sansone V, Da Gama MM (2003) Result of arthroscopic treatment of pigmented villonodular synovitis of the knee. Arthroscopy 19:602-607

23. Chiari C, Pirich C, Brannath W, Kotz R, Trieb K (2006) What affects the recurrence and clinical outcome of pigmented villonodular synovitis? Clin Orthop Relat Res 450:172-178

24. Wu CC, Pritsch T, Bickels J, Wienberg T, Malawer MM (2007) Two incision synovectomy and radiation treatment for diffuse pigmented villonodular synovitis of the knee with extra-articular component. Knee 14:99-106

25. Dines JS, DeBerardino TM, Wells JL, Dodson CC, Shindle M et al (2007) Long-term follow-up of surgically treated localized pigmented villonodular synovitis of the knee. Arthroscopy 23: 930-937

26. Ozturk H, Bulut O, Oztemur Z, Bulut S (2008) Pigmented villonodular synovitis managed by Yttrium 90 after debulking surgery. Saudi Med J 29:1197-1200

27. Nassar WA, Bassiony AA, Elghazaly HA (2009) Treatment of diffuse pigmented villonodular synovitis of the knee with combined surgical and radiosynovectomy. HSS J 5:19-23

28. Liu C, Zhao J, Chen L (2009) Clinical results of arthroscopic treatment for localized pigmented villonodular synovitis of knee. Zhongguo Xiu Fu Chong Jian Wai Ke Za Zhi 23:1042-1044

29. Akinci O, Akalin Y, Incesu M, Eren A (2011) Long-term results of surgical treatment of pigmented villonodular synovitis of the knee. Acta Orthop Traumatol Turc 45:149-155

30. Griffin AM, Ferguson PC, Catton CN, Chung PW, White LM et al (2012) Long-term outcome of the treatment of high-risk tenosynovial giant cell tumor/pigmented villonodular synovitis with radiotherapy and surgery. Cancer 118:4901-4909

31. Nakahara H, Matsuda S, Harimaya K, Sakamoto A, Matsumoto Y et al (2012) Clinical results of open synovectomy for treatment of diffuse pigmented villonodular synovitis of the knee: case series and review of literature. Knee 19:684-687

32. Loriaut P, Djian P, Boyer T, Bonvarlet JP, Delin C et al (2012) Arthroscopic treatment of localized pigmented villonodular synovitis of the knee. Knee Surg Sports Traumatol Arthrosc 20:1550 1553

33. van der Heijden L, Mastboom MJ, Dijkstra PD, van de Sande MA (2014) Functional outcome and quality of life after the surgical treatment for diffuse-type giant-cell tumour around the knee: a retrospective analysis of 30 patients. Bone Joint J 96-B:1111-1118

34. Jain JK, Vidyasagar JV, Sagar R, Patel H, Chetan ML, Bajaj A (2013) Arthroscopic synovectomy in pigmented villonodular synovitis of the knee: clinical series and outcome. Int Orthop 37(12): 2363-9

35. Ma X, Shi G, Xia C, Liu H, He J, Jin W (2013) Pigmented villonodular synovitis: a retrospective study of seventy five cases (eighty one joints). Int Orthop 37(6):1165-70

36. Botez P, Sirbu PD, Grierosu C, Mihailescu D, Savin L, Scarlat MM (2013) Adult multifocal pigmented villonodular synovitis-clinical review. Int Orthop 37(4):729-33

37. Valdez RS, Guterbock TM (2014) Beyond traditional advertisements: leveraging facebook's social structures for research recruitment. J Med Internet Res 16:e243

38. Vastel L, Lambert P, De PG, Charrois O, Kerboull M et al (2005) Surgical treatment of pigmented villonodular synovitis of the hip. J Bone Joint Surg Am 87:1019-1024

39. Hamlin BR, Duffy GP, Trousdale RT, Morrey BF (1998) Total knee arthroplasty in patients who have pigmented villonodular synovitis. J Bone Joint Surg Am 80:76-82

40. Ogura K, Yasunaga H, Horiguchi H, Ohe K, Shinoda Y et al (2013) Impact of hospital volume on postoperative complications and inhospital mortality after musculoskeletal tumor surgery: analysis of a national administrative database. J Bone Joint Surg Am 95:16841691

41. Slevin ML, Plant H, Lynch D, Drinkwater J, Gregory WM (1988) Who should measure quality of life, the doctor or the patient? Br J Cancer 57:109-112

42. Scarlat MM, Mavrogenis AF, Pecina M, Niculescu M (2015) Impact and alternative metrics for medical publishing: our experience with international orthopaedics. Int Orthop 39: $1459-1464$ 